\title{
The Proposal of the Activities of Daily Living (ADL) Index for Institutionalized Older Adults.
}

\author{
Shinichi DEMURA*1, Susumu SATO*2, Masaki MINAMI*3, \\ Hidetsugu KOBAYASHI ${ }^{* 4}$ and Masahiro NODA*5 \\ *' Kanazawa University, Faculty of Education, Kakuma, Kanazawa, Ishikawa \\ *2 Kanazawa Institute of Technology, Ohgigaoka, Nonoichi, Ishikawa \\ *3 Kanazawa college of art, Kodatsuno, Kanazawa, Ishikawa \\ ${ }^{*}$ Fukui National College of Technology, Geshi, Sabae, Fukui \\ *' Jin-ai Women's College, Amaike, Fukui, Fukui
}

\begin{abstract}
The purpose of this study was to propose the ADL index constructed with a unidimensional scale based on item difficulty for institutionalized older adults. Six hundreds and three subjects (159 males and 444 females) were divided into the following four groups based on assisting devices for movement: G1 did not use assisting devices for movement; G2 used a stick or a walker; G3 used a wheelchair; G4 could not move. As the results of examinations from the points of the approximation of proportions, non-answer rates and agreement rates of each item according to $74 \mathrm{ADL}$ items representing $9 \mathrm{ADL}$ domains, the $27 \mathrm{ADL}$ items were selected as utility items. The reliability and unidimensionality of the ADL index consisting of 27 items were considered to be high. As the results of examining the difficulty of items and ADL ability characteristics of each group, the ADL concerning movement and other lower limb activity in G1 and G2, and concerning changes of posture and manual activity in G3 and G4 were considered to reflect the individual differences of ADL ability, respectively. The numbers of ADL items which should first be assessed in each group were as follows: 20 items in G1; 21 items in G2; 21 items in G3; 11 items in G4.
\end{abstract}

Jpn J Hyg 2000; 55: 538-46

\section{Effects of low concentration of styrene monomer vapor on pregnancy}

\author{
Ruriko NINOMIYA*1, Yoshiko HIROKAWA*2, Ryoji YAMAMOTO*1, Hidehisa MASUI*', \\ Naoko KOIZUMI*1 and Akira KUBOTA*3 \\ *' Department of Public Health, Hyogo College of Medicine, Hyogo \\ *2 School of Design and Architecture, Nagoya City University, Nagoya \\ *3 Department of Surgical Pathology, Hyogo College of Medicine, Hyogo
}

Summary To investigate the effects of exposure to styrene vapor on pregnancy, mice proved to be pregnant were exposed to $0,2,20$ and $100 \mathrm{ppm}$ styrene continuously for 24 hours during the period from day 0 to day 15 of gestation. A special small exposure chamber which can accurately maintain a constant low concentration of styrene vapor was made and used in the experiment.

1) In the $100 \mathrm{ppm}$ group, hyperkinesis was presented in the chamber during the exposure period and inhibition of body weight gain was found $(\mathrm{p}<0.01)$. No deaths occurred in any of the groups.

2) At necropsy of dams, no significant difference was found between the styrene exposed groups and the control group in any of the fertility indices, number of implantations, number of live fetuses, percentage of resorptions, or in the percentage of dead fetuses. The $100 \mathrm{ppm}$ group showed lower fetal and placental weights $(\mathbf{p}<0.01)$.

3) Gross necropsy of dams in the $100 \mathrm{ppm}$ group showed significantly decreased adipose tissue. The liver, kidney and spleen weights were also low $(\mathrm{p}<0.01)$.

4) When exposed to $100 \mathrm{ppm}$, which corresponds to 5 times the permissible concentration, i.e., $20 \mathrm{ppm}$, for 24 hours, non-pregnant dams showed no abnormality, while pregnant dams showed inhibition of body weight gain and significantly lower fetal and placental weights and maternal organ weights. Therefore, careful consideration should be given to exposure of women of childbearing age to styrene. 


\title{
A Study on the Trend of Tuberculosis in an Area of Shanghai City Using Mathematical Model
}

\author{
Yuanyao FANG \\ Department of Epidemiology and Environmental Health Juntendo University School of Medicine, Tokyo
}

\begin{abstract}
A widely-used mathematical model (compartmental model) in epidemiological studies was utilized to analyze the tuberculosis situation in area $\mathrm{Y}$ of Shanghai from 1971 till 1994. In this model, the whole population was divided into 5 groups, (susceptible, latent, prevalent, healed and immune). Six parameters (effective BCG coverage, immune loss rate, infection rate, disease risk, cure rate and heal loss rate) were introduced and discussed to describe the transmission of tuberculosis. The computed tuberculosis data from this model agree quite well with the observed data. The results show that the susceptible group is the largest among all groups. In 1994, the annual risk of infection was estimated to be $0.26 \%$. It is predicted that elderly people will comprise an increasing percentage of tuberculosis patients in the future. It is also found that it will still take a long time to eliminate tuberculosis under current medical situations.
\end{abstract}

Jpn J Hyg 2000; 55: 552-8

\section{Smoking Behaviors among Medical Doctors in Fukui, Japan.}

\author{
Takashi OHIDA*1, Kazuo KAWAHARA*2, Yoneatsu OSAKI*3, Tomofumi SONE*1, Tosihiro ISHII*1, \\ Tetsuya FUJIMORI*4, Takeshi KAWAGUCHI ${ }^{* 5}$ and Masumi MINOWA*6 \\ *'Department of Public Health Administration, National Institute of Public Health, Tokyo \\ *2Department of Health Policy Science, Tokyo Medical and Dental University Grand School of \\ Medical and Dental Science, Tokyo \\ *.3 Department of Hygiene, Faculty of Medicine, Tottori University, Tottori \\ * Department of Preventive and Environmental Health, Osaka City University, Medical School, Osaka \\ *'Department of Public Health, Showa University School of Medicine, Tokyo \\ ${ }^{*}$ Department of Epidemiology, National Institute of Public Health, Tokyo
}

\begin{abstract}
This study was conducted in order to identify the smoking prevalence among all doctors $(\mathrm{N}=874)$ belonging to the medical associations in Fukui Prefecture and factors that might possibly indicate causal smoking habits. A survey was conducted from December of 1996 to February of 1997, using a self-administered questionnaire, and the response rate for the survey was $91 \%$. The main results of this survey were as follows: The prevalence of smoking among male and female medical doctors was $28 \%$ and $8 \%$ respectively, which was lower than that of adults in the general population, but higher than that of doctors in the developed countries. The prevalence of smoking among doctors was almost highest when they were 20 to 29 years old, and that among medical practitioners with their own institution was higher than doctors employed by an institution. In particular, medical practitioners who did not smoke were more likely to restrict or ban smoking in their hospitals or clinics.
\end{abstract}

Jpn J Hyg 2000; 55: 559-65 